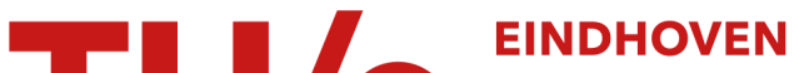 \\ UNIVERSITY OF \\ TECHNOLOGY
}

\section{Flow anisotropy in rotating buoyancy-driven turbulence}

\section{Citation for published version (APA):}

Rajaei, H., Joshi, P. R., Kunnen, R. P. J., \& Clercx, H. J. H. (2016). Flow anisotropy in rotating buoyancy-driven turbulence. Physical Review Fluids, 1(4), [044403]. https://doi.org/10.1103/PhysRevFluids.1.044403

DOI:

10.1103/PhysRevFluids.1.044403

Document status and date:

Published: 01/08/2016

\section{Document Version:}

Accepted manuscript including changes made at the peer-review stage

\section{Please check the document version of this publication:}

- A submitted manuscript is the version of the article upon submission and before peer-review. There can be important differences between the submitted version and the official published version of record. People interested in the research are advised to contact the author for the final version of the publication, or visit the $\mathrm{DOI}$ to the publisher's website.

- The final author version and the galley proof are versions of the publication after peer review.

- The final published version features the final layout of the paper including the volume, issue and page numbers.

Link to publication

\section{General rights}

Copyright and moral rights for the publications made accessible in the public portal are retained by the authors and/or other copyright owners and it is a condition of accessing publications that users recognise and abide by the legal requirements associated with these rights.

- Users may download and print one copy of any publication from the public portal for the purpose of private study or research.

- You may not further distribute the material or use it for any profit-making activity or commercial gain

- You may freely distribute the URL identifying the publication in the public portal.

If the publication is distributed under the terms of Article 25fa of the Dutch Copyright Act, indicated by the "Taverne" license above, please follow below link for the End User Agreement:

www.tue.nl/taverne

Take down policy

If you believe that this document breaches copyright please contact us at:

openaccess@tue.nl

providing details and we will investigate your claim. 


\title{
Flow anisotropy in rotating buoyancy-driven turbulence
}

\author{
Hadi Rajaei,* Pranav Joshi, Rudie P. J. Kunnen, and Herman J. H. Clercx \\ Fluid Dynamics Laboratory, Department of Applied Physics and J. M. Burgers Center for Fluid Dynamics, \\ Eindhoven University of Technology, P.O. Box 513, 5600 MB Eindhoven, The Netherlands
}

\begin{abstract}
We report a combined experimental-numerical study of the effects of background rotation on large- and small-scale isotropy in rotating Rayleigh-Bénard convection (RBC) from both Eulerian and Lagrangian points of view. 3D particle tracking velocimetry (3D-PTV) and direct numerical simulations (DNS) are employed at three different heights within the cylindrical cell. The Lagrangian velocity fluctuation and second-order Eulerian structure function are utilized to evaluate the large-scale isotropy for different rotation rates. Furthermore, we examine the experimental measurements of the Lagrangian acceleration of neutrally buoyant particles and the second-order Eulerian structure function to evaluate the small-scale isotropy as a function of rotation rate. It is found that background rotation enhances large-scale anisotropy at the cell center and close to the top plate, while decreases it at intermediate height. The large-scale anisotropy, induced by rotation, has negligible effect on the small scales at the cell center, whereas the small scales remain anisotropic close to the top plate.
\end{abstract}

\section{INTRODUCTION}

Kolmogorov's hypothesis of local isotropy states that the turbulent flow statistics should be universal at sufficiently high Reynolds numbers and small scales; they are independent of the large-scale flow structure. According to the hypothesis, the information of the preferred direction of the large-scale flow is lost during the cascade from large to small scales. Several numerical and experimental studies address the effects of large-scale anisotropy on the small scales for different types of flow. Contrary to Kolmogorov's hypothesis of local isotropy, small-scale anisotropy is reported in the flow between two counterrotating disks [1], shear flow [2,3], channel flow [4], and turbulent shear-less mixing [5]. Since the majority of simulations and models rely on the assumption of local isotropy and all turbulent flows in nature and technological applications suffer from large-scale anisotropy, the study of the effects of large-scale anisotropy on small scales plays a major role in our understanding of these flows.

In the present investigation, we focus on large- and small-scale anisotropy induced by background rotation in a classical system to study buoyancy-driven flows, Rayleigh-Bénard convection (RBC). RBC is the motion of a fluid confined between horizontal plates, heated from below and cooled from above. Buoyancy-driven flow subjected to rotation is ubiquitous in nature (e.g. the effects of Earth's rotation on oceanic [6-8] and atmospheric currents [9]) and in technological applications (e.g. convective cooling in turbomachinery $[10,11])$. This explains why rotating $\mathrm{RBC}$ has been extensively studied in laboratory experiments, e.g. Refs. [12-25], and simulations, e.g. Refs. [22, 26-32]. A recent review paper on this topic is Ref. [33].

It has been reported earlier in Ref. [18] that the

\footnotetext{
*h.rajaei@tue.nl
}

background rotation in RBC amplifies the large-scale anisotropy in the bulk while it reduces anisotropy closer to the plate at height $z=0.8 H$ ( $H$ is the height of the convection cell). In the present work, we first analyze the effects of background rotation on the largescale anisotropy at three different heights in rotating RBC. Then we focus on the effects of rotation-induced large-scale anisotropy on the small scales. We use a combined experimental-numerical approach to investigate the small-scale anisotropy from Eulerian and Lagrangian viewpoints.

The remainder of the paper is organized as follows. The experimental and numerical setups are discussed in Sec. II. We start with a brief introduction on the flow phenomenology in Sec. III A. In Sec. III B, the results on large-scale (an)isotropy are discussed based on velocity statistics and the inertial range of the second-order Eulerian structure function. Next, we discuss the results on small-scale (an)isotropy in Sec. IIIC based on the dissipative range of the second-order Eulerian SF and acceleration statistics. We summarize our main findings in Sec. IV.

\section{METHODS AND PARAMETERS}

\section{A. Experimental setup}

The experimental setup consists of a convection cell, similar to the one used in [21, 24], and a particle tracking system based on the system developed at ETH Zürich (Switzerland) [34-38]. Although the details of the setup and particle tracking methodology can be found in the references, we briefly summarize the most important aspects below.

A schematic view of the experimental setup is presented in Fig 1(a). The convection cell is composed of three main parts; (I) a heated plate at the bottom, (II) a cooling chamber at the top, and (III) a cylindrical Plexiglas vessel connecting top and bottom plates, see Fig 

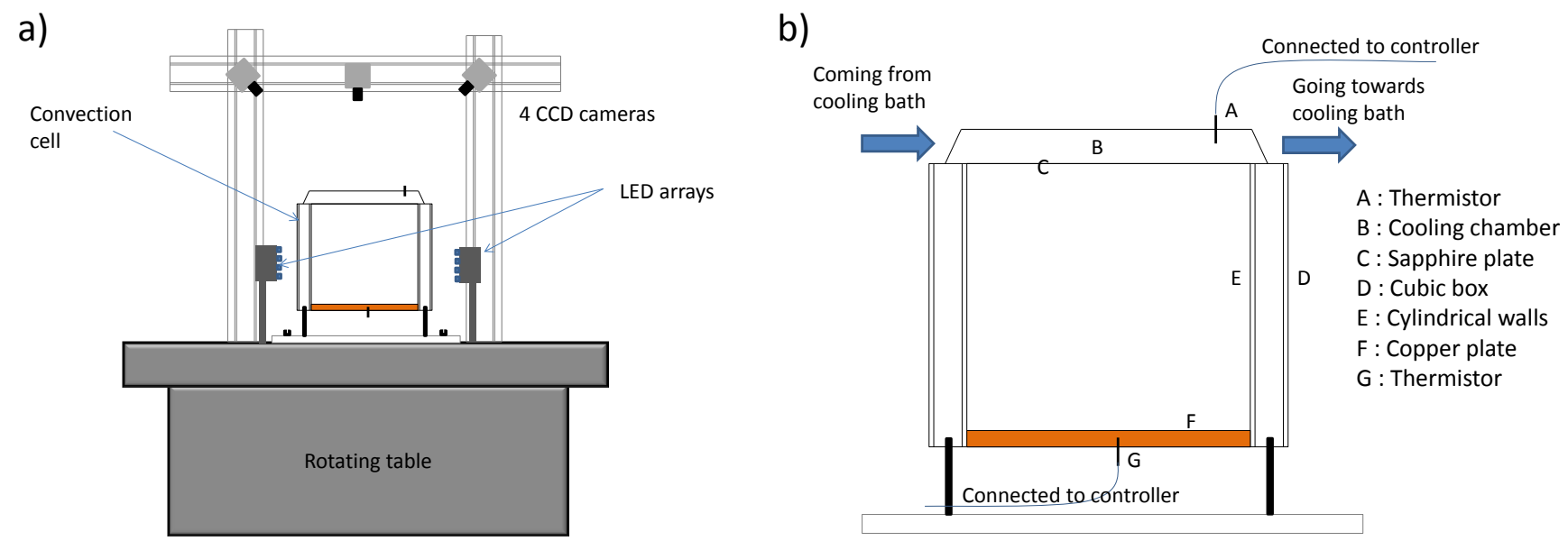

FIG. 1. (a) Schematic view of the experimental set-up and (b) the convection cell and its components.

1(b). At the bottom, an electrical resistance heater is attached to a copper plate; its temperature is measured by a thermistor placed inside a hole close to the wetted surface at the center of the copper plate. The cooling chamber is placed at the top of the convection cell. A sapphire plate is positioned at the bottom of the cooling chamber, guaranteeing good thermal contact between working fluid and the recirculating fluid in the cooling bath while retaining transparency. A Plexiglas plate is located on the top of the cooling chamber. The cooling chamber is transparent and as a result it makes the cell optically accessible from above. The cylindrical Plexiglas vessel with equal inner diameter $D$ and height $H$ of $200 \mathrm{~mm}$, filled with water, is placed inside a rectangular Plexiglas box. The space between cylinder and box is filled with water as well to avoid the distortion of the illumination from the side.

The particle tracking system consists of four chargecoupled device (CCD) cameras (MegaPlus ES2020, $1600 \times 1200$ pixels $)$ positioned above the convection cell, see Fig 1(a). The cameras record the flow field at a frequency of $30 \mathrm{~Hz}$. This is adequate to resolve the smallest length and time scales of the flow. The recorded images are transferred to a computer simultaneously which amounts to a data transfer rate of $240 \mathrm{MB} / \mathrm{s}$.

In these experiments fluorescent polyethylene particles (density $\rho_{p}=1002 \mathrm{~kg} / \mathrm{cm}^{3}$, diameter $d_{p}=75-90 \mu \mathrm{m}$, thermal conductivity $\lambda=0.5 \mathrm{~W} /(\mathrm{m} \mathrm{K}))$ are used as tracer particles. The ratio between particle response time and Kolmogorov time scale is expressed by the Stokes number St $=\tau_{p} / \tau_{\eta}$, where $\tau_{p}=d_{p}^{2}\left(\rho_{p}-\rho_{f}\right) /\left(18 \nu \rho_{f}\right)$ is the so-called particle response time, $\nu$ the kinematic viscosity of the fluid, $\rho_{f}$ the fluid density and $\tau_{\eta}$ is the Kolmogorov time scale. For the present experiments, the Stokes number is small, St $\approx 2 \times 10^{-6}$; the particles can be considered tracers.

The illumination is provided by four arrays of light- emitting diodes (LEDs), operated in pulsed mode and triggered by the cameras, with a dominant wavelength of $455 \mathrm{~nm}$. The fluorescent particles emit light at wavelength of $600 \mathrm{~nm}$ which provides a sufficient upshift with respect to the illumination wavelength, resulting in easy separation of direct and fluorescent light. The cameras use high-pass filters (OG-570, Schott Glass) which filter out the spurious reflections with a wavelength of $455 \mathrm{~nm}$.

\section{B. Experimental parameters}

Four non-dimensional parameters are important in rotating RBC, namely the Rayleigh number Ra $=\alpha g \Delta T H^{3} /(\kappa \nu)$, Prandtl number $\operatorname{Pr}=\nu / \kappa$, Rossby number Ro $=\sqrt{\alpha g \Delta T / H} /(2 \Omega)$ and aspect ratio $\Gamma=D / H$, where $g$ is the gravitational acceleration, $\Delta T$ the applied temperature difference, $\Omega$ the rotation rate, and $\alpha$ and $\kappa$ are the thermal expansion coefficient and thermal diffusivity of the fluid, respectively. In the present study $\mathrm{Ra}=1.28 \times 10^{9}, \mathrm{Pr}=6.7$ and $\Gamma=1$ are the fixed parameters while Ro varies between 0.1 and $\infty$ ( $\Omega$ between $1.65 \mathrm{rad} / \mathrm{s}$ and 0 ). The mean temperature is set to $22^{\circ} \mathrm{C}$ and $\Delta T=10^{\circ} \mathrm{C}$.

The velocity fluctuation in the $i$ direction is defined as

$$
u_{i}=\left\langle\left(U_{i}-\left\langle U_{i}\right\rangle\right)^{2}\right\rangle^{1 / 2}
$$

where $U_{i}$ is the $i^{\text {th }}$ component of the velocity signal and $\langle\ldots\rangle$ indicates the time average. Note that the statistics in $x$ and $y$ directions are almost the same, i.e. $u_{x} \cong u_{y}$. Thus, we only present the values in one direction $(x$ direction) and refer to it as the horizontal component, $u_{h}$. Likewise, the acceleration fluctuation in the $i$ direction is defined as

$$
a_{i}=\left\langle\left(A_{i}-\left\langle A_{i}\right\rangle\right)^{2}\right\rangle^{1 / 2}
$$


where $A_{i}$ is the $i^{\text {th }}$ component of the acceleration signal and $\langle\ldots\rangle$ indicates the time average defined as above. Similar to the velocity fluctuation, the horizontal component of the acceleration fluctuation is represented by $a_{x}$ and we refer to it as $a_{h}$.

In this paper, we discuss the measurements in three different regions. Region I is a volume of $50 \times 50 \times$ $50 \mathrm{~mm}^{3}(x, y, z)$, which is located in the center of the cylinder thus covering the height $0.375 H<z<0.625 H$. Region II is a volume of $50 \times 50 \times 20 \mathrm{~mm}^{3}(x, y, z)$ placed between $z=0.75 H$ and $z=0.85 H$ with its center on the cylinder axis. Region III is close to the top plate, a volume of $50 \times 50 \times 10 \mathrm{~mm}^{3}(x, y, z)$ with its center on the cylinder axis covering the height $0.95 H<z<H$. For the remainder of the paper, we shall refer to these locations by the vertical position of their center, i.e. $z=0.5 \mathrm{H}$ (center), $z=0.8 H$ and $z=0.975 H$, respectively. The statistics are based on approximately $7 \times 10^{7}$ data points at the center and $z=0.8 H$ and approximately $3.5 \times 10^{7}$ data points for $z=0.975 H$. Note that the kinetic boundary layer at the top plate has a thickness between 1.6 and $6 \mathrm{~mm}$, depending on Ro [39]. Therefore, between 16 and $60 \%$ of the upper measurement volume at $z=0.975 H$ is occupied by the kinetic boundary layer. The duration of the experiment for each rotation rate is about 300 minutes (consisting of multiple segments, each 11 minutes long).

\section{Numerical setup}

In parallel to the experiments, direct numerical simulations (DNS) are performed. A cylindrical configuration with the same aspect ratio as the experiment $(\Gamma=1)$ is realized in simulations, where for convenience the discretization of the equations of motions is done in cylindrical coordinates. The boundary conditions are no-slip at all walls, isothermal plates on top and bottom (bottom plate temperature $\Delta T$ higher than top), and an adiabatic sidewall. Details of the numerical method can be found in Refs. [21, 40, 41]. The number of grid points in radial, azimuthal and vertical directions is 513, 257, and 513, respectively. Grid refinement is applied close to the side wall, bottom and top boundaries. The resolution is checked a posteriori by validating that the Kolmogorov scale is resolved and there is an adequate number of grid points within the boundary layers (at least 26 grid points).

\section{RESULTS}

In this section, we first discuss the flow phenomenology. Then, the effects of background rotation on large scales are discussed. Later on, the small-scale (an)isotropy is examined.

\section{A. Flow phenomenology}

Non-rotating Rayleigh-Bénard convection is characterized by a domain-filling large-scale circulation (LSC) [42]. The LSC results in mean vertical velocities near the side wall and mean horizontal velocities near the top and bottom plates. Fig. 2(a) shows a few trajectories from the experiments initiated at the same time for $\operatorname{Ro}=\infty$. Fig. 2(b) is a projection of Fig. 2(a) on the horizontal $(x y)$ plane (top view). The red circles indicate the starting points of the trajectories. It is well-known that the LSC exists for large values of $\operatorname{Ro}(\operatorname{Ro} \gtrsim 2.5$ for $\Gamma=1)$ $[43,44]$, and the presence of the resulting non-zero mean velocity is clear in Fig. 2(a) and (b).

However, for lower values of Ro (higher rotation rates) the LSC is washed away and is replaced by vertically aligned vortical plumes $[43,44]$. Fig. 2(c) shows a few particle trajectories commencing at the same time for Ro $=0.1$ and Fig. 2(d) shows their projections on the horizontal plane. The mean velocity field disappears for the high rotation rates. The vortical plumes can be recognized in Fig. 2(d).

\section{B. Large-scale (an)isotropy}

\section{Velocity statistics}

It has been shown in Ref. [18], by using the so-called Lumley map, that rotation enhances the large-scale anisotropy at the cell center, while it reduces anisotropy close to the top plate (based on planar stereo particle image velocimetry measurements at $z=0.8 H$ ). The velocity fluctuation is the velocity scale typical for the largest turbulent scales in the flow. Therefore, anisotropy of the velocity fluctuation indicates anisotropy of the large scales.

As mentioned in Sec. III A, the LSC is the main feature of the flow for $\operatorname{Ro} \gtrsim 2.5$ (for $\Gamma=1$ ) [43, 44]. The presence of the LSC leads to a non-zero mean for horizontal velocity at $z=0.8 H$ and $z=0.975 H$. Therefore, it is required to subtract the mean of the velocity from the velocity signal to obtain the velocity fluctuation. As mentioned earlier, the experiment for each rotation rate consists of multiple segments each approximately 11 minutes long. The mean of the velocity is calculated based on the mean of the velocity for each of these segments. Thus, the time averages in Eq. (1) and (2) are performed on approximately 11 min segments. The mean is calculated for each segment since the LSC orientation changes under rotation: the LSC precesses against the direction of rotation [19, 43, 45]. Therefore, it is pointless to use the mean value of the velocity for the entire duration of the experiments $(\sim 300 \mathrm{~min})$. Note that these subtractions are only applied on the horizontal component of the velocity for $\mathrm{Ro} \geq 2.5$ at $z=0.8 H$ and $z=0.975 H$ where the horizontal velocity possesses a non-zero mean. For Ro $<2.5$ at $z=0.8 H$ and $z=0.975 H$ and for all 
a)

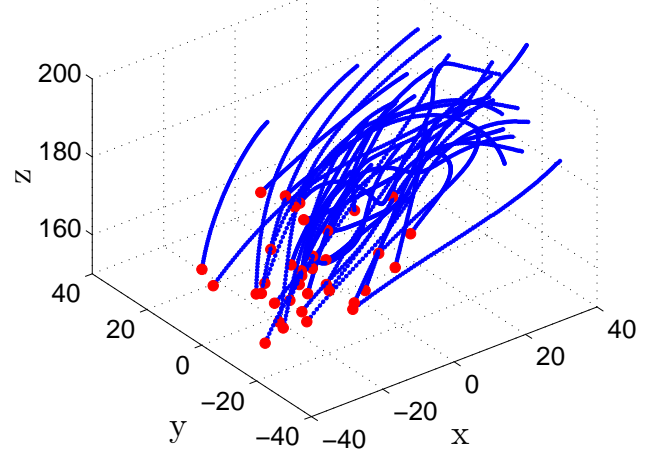

c)

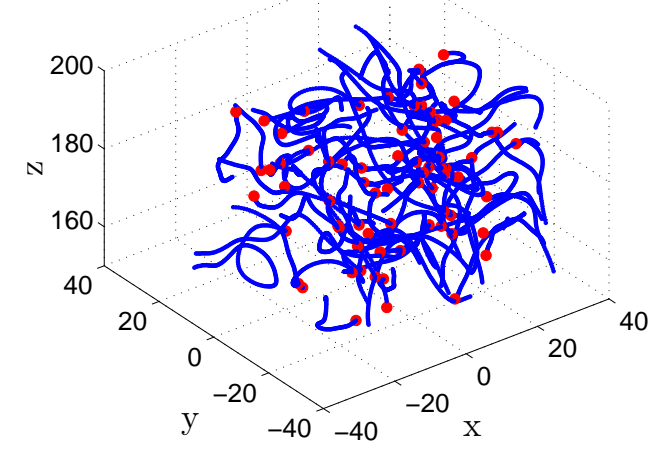

b)

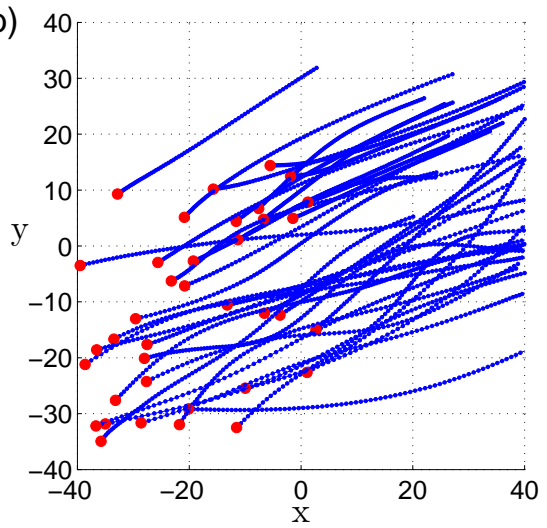

d)

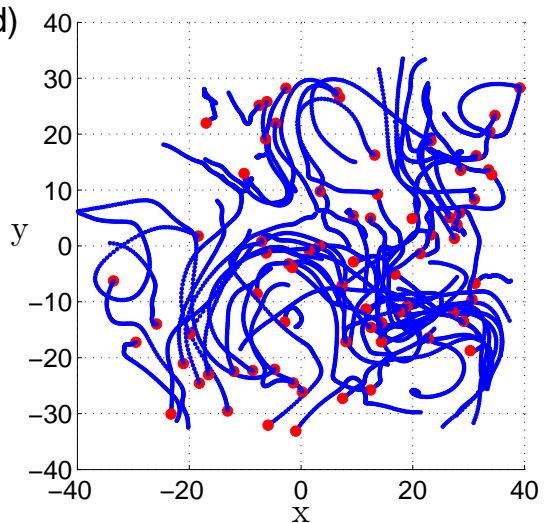

FIG. 2. Particle trajectories from experiments near the top plate for (a) $\operatorname{Ro}=\infty$, isometric view (b) Ro $=\infty$, top view, (c) $\mathrm{Ro}=0.1$, isometric view and (d) Ro $=0.1$, top view. The axes are in $\mathrm{mm}$ and the top plate is located at $z=200 \mathrm{~mm}$.

measured Ro at the cell center, as for the vertical velocity in all cases, the velocity has a zero mean and thus no averaging is used.

It is worth mentioning that the calculated mean velocity value depends on the duration of the averaging. A shorter averaging time results in a truncation of the low frequency fluctuations and thus an underestimation of the velocity fluctuation. Therefore, the uncertainty in the velocity fluctuation for Ro $\geq 2.5$ at $z=0.975 H$ and $z=0.8 H$ is higher compared to other data points.

Fig. 3(a) shows the velocity fluctuations as a function of Ro for the three measurement sets. The results from the current Lagrangian investigation are in good qualitative agreement with earlier measurements [21] using stereoscopic particle image velocimetry (an Eulerian velocity measurement) in a similar system at comparable Ro, Ra and Pr. As can be seen from the graph, at the center $(z=0.5 H)$ the vertical and horizontal velocity fluctuations (blue squares and circles, respectively) are approximately equal for Ro $=\infty$ (non-rotating case). The vertical component is significantly enhanced under weak rotation $(2.5 \leq$ Ro $\leq 5)$. A similar but much weaker trend is observed for its horizontal counterpart. For Ro $\lesssim 2.5$, both horizontal and vertical components decrease with decreasing Ro due to the suppression of turbulence intensity by the background rotation. The horizontal and vertical velocity fluctuations scale as $u \sim \operatorname{Ro}^{0.22}$ ( $u$ can be either horizontal or vertical component of the velocity) at the center for Ro $\leq 2.5$; similar scaling has been reported for the Eulerian velocity at comparable Ra and $\operatorname{Pr}, u \sim \mathrm{Ro}^{0.2}[21]$. The ratio $R=u_{z} / u_{h}$, plotted in Fig. 3 (b), can be used as a measure of (an)isotropy of the large-scale velocity fluctuations. The ratio $R$ at the cell center, blue squares in Fig. 3 (b), shows clearly that the large-scale anisotropy in the velocity field increases with decreasing Ro.

For $2.5 \leq$ Ro $\leq 5$, measurements of the velocity fluctuation values at $z=0.8 H$, cyan triangles and stars in Fig. 3(a), show similar behaviour as the measurements at the cell center; a significant and a slight increase in vertical and horizontal components, respectively. For Ro $<2.5$ both vertical and horizontal velocity components decrease with increasing rotation rate, similar to those from the cell center measurements. However, reduction of the vertical component is higher than that of the horizontal one. This is clear from the anisotropy ratio $R$, Fig. 3(b), which approaches one with decreasing Ro. For Ro $>2.5$, the large-scale anisotopy seems to increase 
from non-rotating to weakly rotating $\mathrm{RBC}$ (but note that there is higher uncertainty for Ro $>2.5$ ). It can be concluded that large-scale anisotropy decreases with increasing rotation rate at $z=0.8 H$ for $\mathrm{Ro} \leq 2.5$. Our findings for measurements at the center and at $z=0.8 \mathrm{H}$ are consistent with earlier reported results in Refs. [18, 21] based on Eulerian velocity measurements.

At $z=0.975 H$, the vertical and horizontal velocity fluctuations are approximately constant for $\mathrm{Ro} \gtrsim 2.5$. The horizontal and vertical velocity fluctuations decrease with reduction in Ro for $\mathrm{Ro}<2.5$. The anisotropy ratio $R$ decreases with decrease in Ro (for Ro $\leq 5$ ) and approaches 0.4 for the lowest Ro (highest rotation rate) in our experiment which indicates that large-scale anisotropy increases with decrease in Ro at $z=0.975 H$.

For the measurements at $z=0.975 H$ (and focusing on Ro $\lesssim 2.5$ ), the horizontal velocity is larger than the vertical one, which is consistent with the presence of strong vortical plumes that add swirling motion in the horizontal plane [18]. However, for the measurements at the cell center, the vertical component is larger than the horizontal one which is an indication that the vortical plumes spin down (become weaker) as they reach the center. The transition between stronger large-scale horizontal motions at the top and stronger large-scale vertical motions at the cell center occurs at $z \approx 0.8 H$.

In conclusion, we have shown that the large-scale anisotropy increases with increase of rotation rate both at the center and near the plate $(z=0.975 H)$. However, the nature of the anisotropy at the center and near the top plate $(z=0.975 H)$ are opposite in the sense that at the center the vertical large-scale motions are stronger while at $z=0.975 H$ the horizontal large-scale motions are stronger. At $z=0.8 H$, the large-scale anisotropy seems to decrease with increasing rotation.

\section{Second-order Eulerian structure function in the inertial range}

It has been shown that the second-order Eulerian structure function (SF), defined as $D(r)=$ $\left\langle(u(x+r)-u(x))^{2}\right\rangle$ with $r$ the magnitude of separation, can be satisfactorily obtained from 3D-PTV data $[46,47]$. In this case, using 3D-PTV data, $D(r)$ is calculated based on randomly distributed particles which requires the assumption of isotropy and homogeneity in the flow field. It might therefore serve as a test of how well isotropy is satisfied. The dissipation rate can be calculated in homogeneous and isotropic turbulence (HIT) based on the second-order Eulerian SF in both inertial $(\eta \ll r \ll \mathcal{L})$ and dissipative $(r<\eta)$ ranges, with $\eta$ the Kolmogorov length scale and $\mathcal{L}$ the integral length scale. In this section, we focus on the equations valid for the inertial range of HIT to provide evidence of (an)isotropy at these scales. Anisotropy at inertial scales implies anisotropy at large scales since it is supposed that the anisotropy is introduced at the large scales.
Within the inertial range of HIT, turbulent flow characteristics can be defined by the dissipation rate alone. The following equations relate the second-order Eulerian $\mathrm{SF}$ in the inertial range to the dissipation rate:

$$
\begin{gathered}
D_{L L}(r)=C_{2}(\epsilon r)^{2 / 3}, \\
D_{N N}(r)=\frac{4}{3} C_{2}(\epsilon r)^{2 / 3},
\end{gathered}
$$

where $D_{L L}(r)$ is the second-order Eulerian longitudinal $\mathrm{SF}, \epsilon$ is the dissipation rate, $C_{2}$ is the universal Kolmogorov constant with $C_{2} \approx 2.13$ [48], $r$ is the magnitude of separation, and $D_{N N}(r)$ is the second-order Eulerian transversal SF. $D_{L L}(r)$ is calculated as follows; at each PTV time step, $\left(u_{1}-u_{2}\right)^{2}$ is calculated for each pair of randomly distributed particles with separation $r \pm \delta r$ ( $\delta r$ is the bin size used for the calculations), where $u_{1}$ and $u_{2}$ are respectively the projections of the velocities of the first and second particle along $\mathbf{r}$ ( $\mathbf{r}$ is defined as a unit vector parallel to the line connecting the two particles). $D_{L L}(r)$ is an average over all pairs with the same separation $r \pm \delta r . D_{N N}(r)$ is calculated with the same method where $u_{1}$ and $u_{2}$ are the projections of the velocities of particles along a line perpendicular to $\mathbf{r}$ and $\mathbf{e}$, with e an arbitrarily chosen unit vector here, defined as $\mathbf{e}=(1,1,1) / \sqrt{3}$. Note that in HIT, $u_{1}$ and $u_{2}$ can be any projection perpendicular to $\mathbf{r}$. Therefore, the direction of unit vector $\mathbf{e}$ is not important.

Fig 4(a-b) shows the compensated structure function, calculated as both $\left(D_{L L}(r) / C_{2}\right)^{3 / 2} / r$ and $\left(3 D_{N N}(r) /\left(4 C_{2}\right)\right)^{3 / 2} / r$ with $C_{2}$ as proposed earlier, based on Eqs. (3) and (4), respectively, for Ro $=\infty$ (Fig $4(\mathrm{a}))$ and Ro $=0.1$ (Fig 4(b)) as a function of separation distance at the cell center. It is reported in Ref. [49] that, using this method, this curve should reach a plateau in the inertial range of HIT at a level corresponding to $\epsilon$. However, as can be seen from Fig 4(a-b), no clear plateau is reached in these cases. Fig. $4(\mathrm{a})$, for $\mathrm{Ro}=\infty$, shows an approximate plateau for $D_{N N}$. However, the measured $\epsilon$ based on this plateau $\left(\sim 1.4 \times 10^{-7} \mathrm{~m}^{2} / \mathrm{s}^{3}\right.$ for $\epsilon$ evaluated from $\left.D_{N N}\right)$ is considerably smaller than that of DNS data $\left(\sim 2.6 \times 10^{-7} \mathrm{~m}^{2} / \mathrm{s}^{3}\right.$, see Fig. $6)$. On the other hand, for Ro $=0.1$ a steep decay is observed after the maximum value for $\epsilon$. The lack of a plateau is due to both the large-scale anisotropy and the comparatively low Taylor-based Reynolds numbers $\left(\operatorname{Re}_{\lambda} \sim 39\right.$ for $\operatorname{Ro}=\infty$ and $\operatorname{Re}_{\lambda} \sim 8$ for $\left.\operatorname{Ro}=0.1\right)$ which limits the extent of the inertial scaling range. The Taylor-microscale Reynolds number, $\operatorname{Re}_{\lambda}$, is estimated here using the isotropic relation $\operatorname{Re}_{\lambda}=u^{2} \sqrt{15 / \epsilon \nu}$ with $u=\left(u_{x}+u_{y}+u_{z}\right) / 3\left(u_{x}, u_{y}\right.$ and $u_{z}$ are all three positive numbers, see Sec. II B). Let us consider the non-rotating case. The flow field at the center of RBC for Ro $=\infty$ is reported to be close to homogeneous and isotropic turbulence [50]. However, the measured dissipation rate based on the inertial range of the second-order structure function for $\mathrm{Ro}=\infty$ does not result in the correct value 
a)

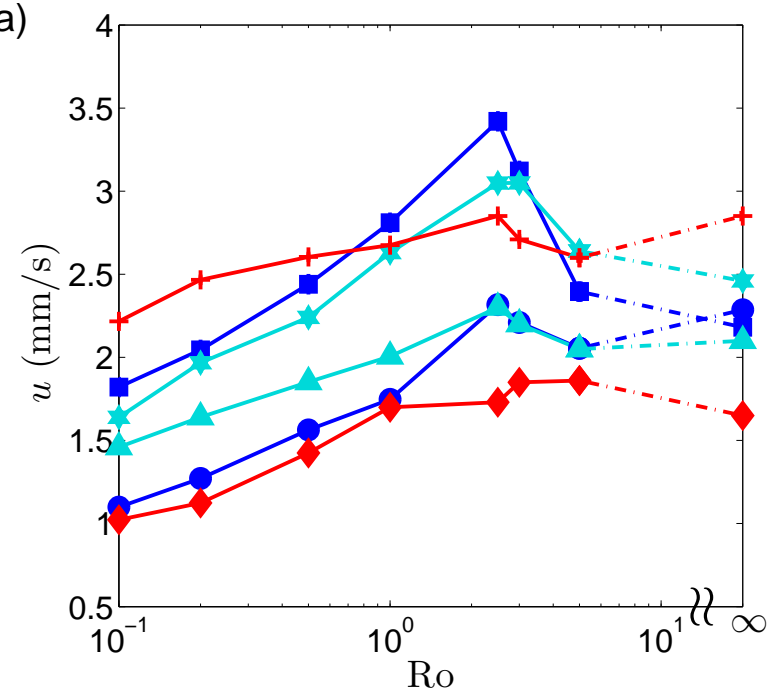

b)

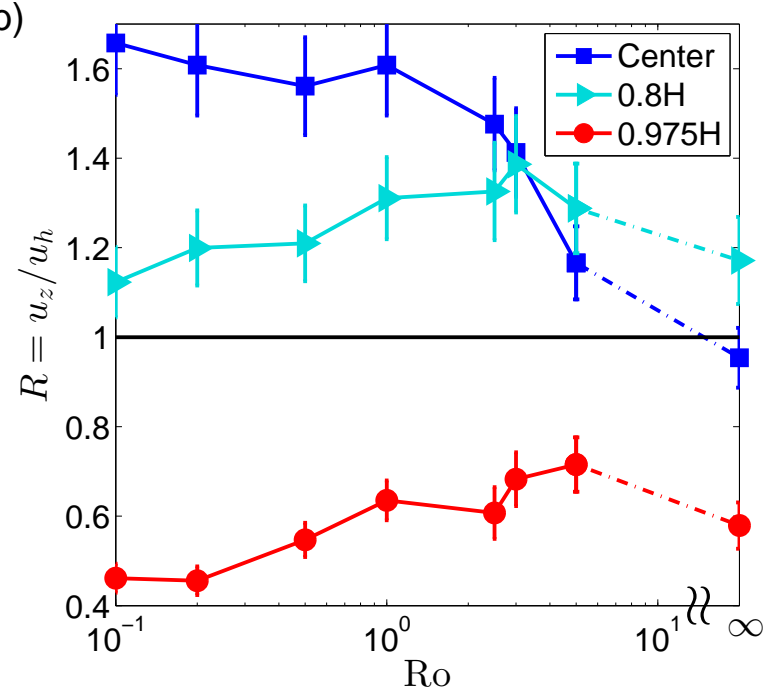

FIG. 3. (a) Horizontal and vertical velocity fluctuations from the experiments. Blue circles and squares are the horizontal and vertical components at the cell center, respectively. Cyan triangles and stars are the horizontal and vertical components at $z=0.8 H$, respectively. Red pluses and diamonds are the horizontal and vertical components at $z=0.975 H$. (b) The anisotropy ratio $R=u_{z} / u_{h}$.

of the dissipation rate. Therefore, it is expected that the limited inertial range is the more direct reason for the absence of a plateau in the non-rotating case (Fig. 4(a)). Fig. 4(b) $($ Ro $=0.1)$ is, nevertheless, different as also anisotropy plays a role. It is worth pointing out that the compensated structure functions for $R o \geq 2.5$ (where the LSC is dominant) are similar to the ones in Fig. 4(a), whereas for Ro $<2.5$ (where the vortical plumes are dominant) the compensated structure functions show a rapid decay as in Fig. 4(b).

The same analysis is done for the measurements at $z=0.8 H$ and $z=0.975 H$ for Ro $\leq 1$ when the mean value of the velocity is zero. A rapid decay, similar to the one in Fig. 4(b), is observed.

It can be concluded that the inertial range (if present at these values of $\operatorname{Re}_{\lambda}$ ) has signs showing anisotropy at all three measurement locations under strong background rotation.

\section{Small-scale (an)isotropy}

\section{Second-order Eulerian structure function in the dissipative range}

Continuing with the analysis of the second-order SF, we test the dissipative range $(r<\eta)$ to measure the compensated structure function and the dissipation rate. At small scales, HIT flow characteristics are determined by the dissipation rate and viscosity. It can be shown [51] that for the dissipative range in HIT the following equations hold:

$$
D_{L L}(r)=\epsilon r^{2} / 15 \nu,
$$

$$
D_{N N}(r)=2 \epsilon r^{2} / 15 \nu .
$$

This method has been used before to calculate the dissipation rate at the cell center in non-rotating RBC [47]. Fig. 5 shows the compensated structure function calculated as both $15 \nu D_{L L}(r) / r^{2}$ and $15 \nu D_{N N}(r) /\left(2 r^{2}\right)$ based on Eqs. (5) and (6), respectively, for Ro $=\infty$ and Ro $=0.1$ at the cell center. The probability of finding a velocity pair with a small separation $r$ is considerably lower than at comparatively larger separation. The scatter of the data at small scales is due to the lack of convergence at these scales. However, the plateau is welldefined. The black lines are fits to the data to determine $\epsilon$. Similar plateaus are observed for the measurements at $z=0.8 H$ and $z=0.975 H$ for all Ro as well. In fact, the Kolmogorov length scale varies between 0.93 and 1.38 $\mathrm{mm}$ in the current experiments. The dissipation-range scaling is valid up to $\sim 10 \eta$ [52]. As can be seen from Fig. 5, the current technique resolves down to $0.4 \mathrm{~mm}$. Therefore, the dissipation range is indeed resolved.

The experimental dissipation rate calculated based on the dissipative range and the dissipation rate calculated directly from DNS are plotted in Fig. 6 for all Ro. The DNS dissipation rate is calculated directly without any assumption of HIT, while the experimental dissipation rate is calculated based on the assumption of HIT at small scales. The comparison shows a very good agreement between DNS and experimental dissipation rate at the cell center, see blue filled and open diamonds. At $z=0.8 H$, the experimental dissipation rates follow the trends of the DNS, while they are generally lower than those from the DNS, see red filled and open circles. Close to the top plate at $z=0.975 H$, neither the trend nor the values match with the DNS dissipation rates, see black 


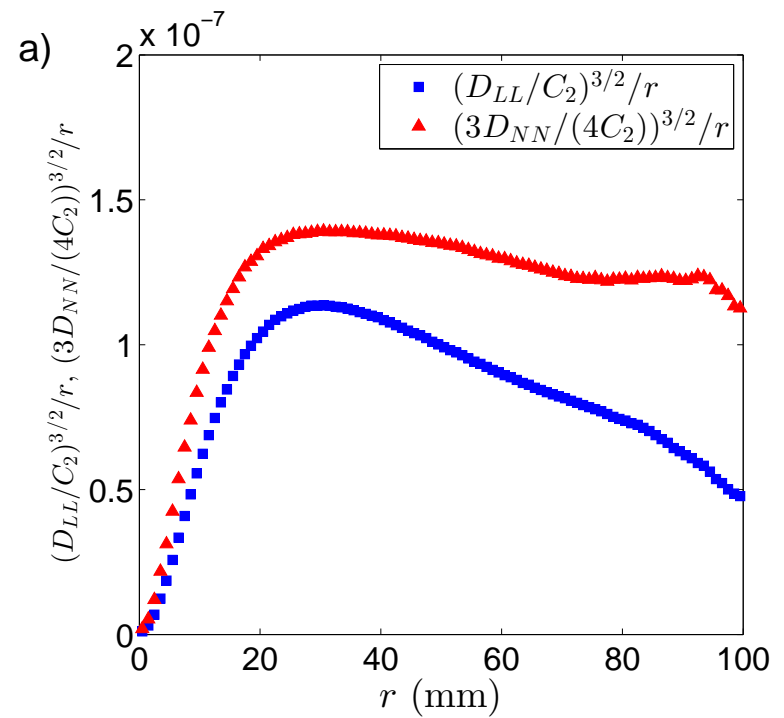

FIG. 4. Compensated structure functions for (a) Ro $=\infty$ and (b) Ro $=0.1$ based on inertial-range scaling at the cell center from the experiments.

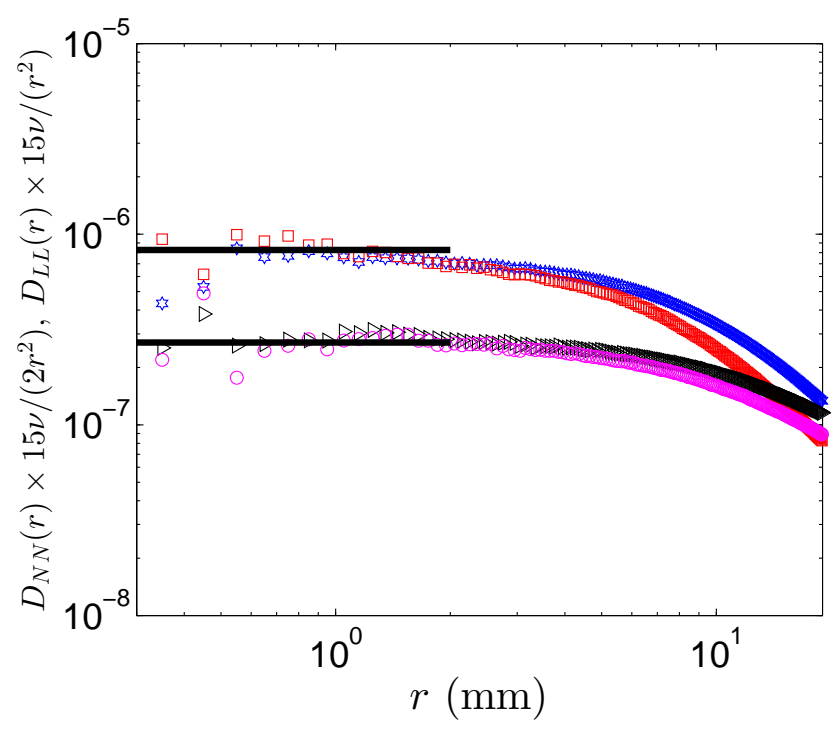

FIG. 5. Compensated structure functions at the cell center for Ro $=\infty$ (pink circles, $D_{N N}(r) \times 15 \nu /\left(2 r^{2}\right)$; black triangles, $\left.D_{L L}(r) \times 15 \nu /\left(r^{2}\right)\right)$ and $R O=0.1$ (red squares, $D_{N N}(r) \times 15 \nu /\left(2 r^{2}\right)$; blue stars, $\left.D_{L L}(r) \times 15 \nu /\left(r^{2}\right)\right)$ based on the dissipative range from the experiments

filled and open squares.

The surprisingly good agreement between DNS and experimental dissipation rate at the cell center indicates that the flow field is indeed homogeneous and isotropic at dissipative scales. At $z=0.8 H$ the velocity fluctuations of the small-scale flow field is not far from isotropy as well, however, at $z=0.975 H$, the assumption of HIT is no longer supported in the dissipative range since the

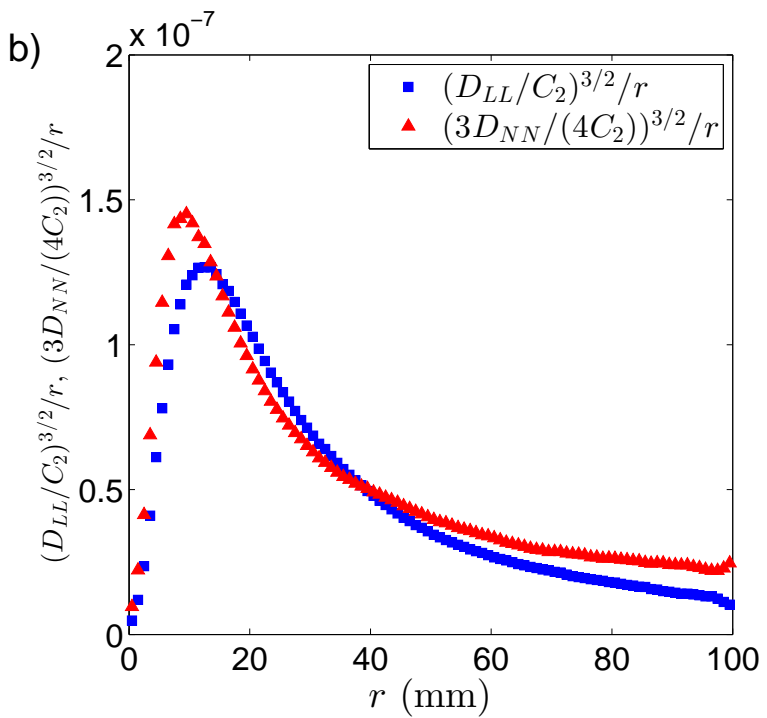
experimental dissipation values are different from DNS data and show a different trend with regard to Ro. In general, the small-scale isotropy deteriorates from center to top, due to the proximity of the wall.

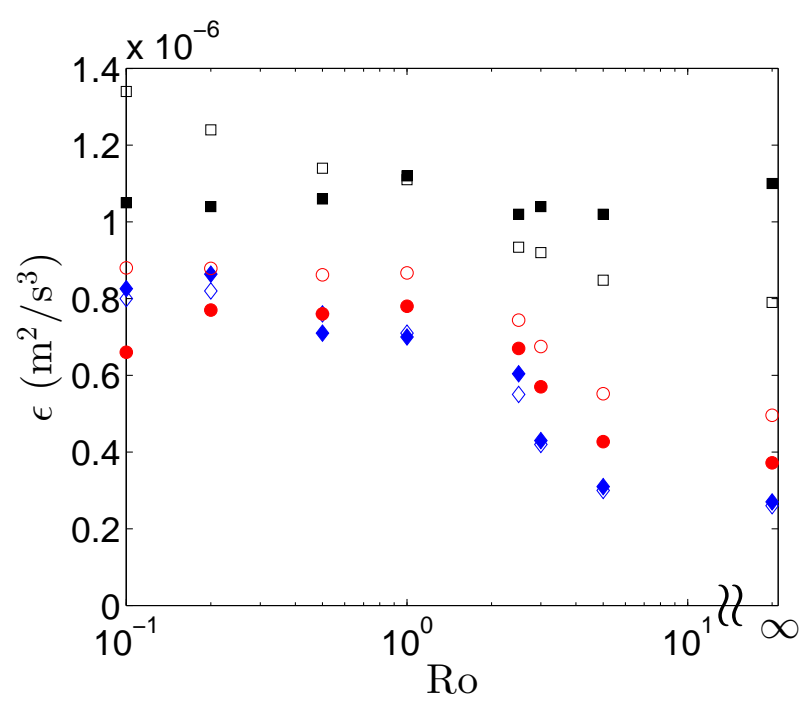

FIG. 6. Dissipation rate for experiment and DNS as a function of Ro. Open symbols are for DNS and filled symbols are for experiments. Blue diamonds, cell center; red circles, at $z=0.8 H$; black squares, at $z=0.975 H$.

\section{Acceleration constant}

In sufficiently small space-time regions in HIT the variance of the Lagrangian acceleration depends only on dis- 
sipation and viscosity. The following equation can be derived [51]:

$$
\left\langle a_{k}^{2}\right\rangle=a_{0} \epsilon^{3 / 2} \nu^{-1 / 2},
$$

where $a_{k}$ is the Lagrangian acceleration component in direction $k$ and $a_{0}$ is a universal constant. The above equation was derived by Heisenberg [53] and Yaglom [54], and is usually referred to as the Heisenberg-Yaglom relation. It is known that the viscosity influences only the small-scale flow motions for large Re [51]. Furthermore, the acceleration scale of the disturbances of length scale $l$ increases with decreasing $l$. Therefore, it can be concluded that Lagrangian acceleration is determined largely by small-scale flow motions $(r<\eta)$ [51]. Thus, we use the acceleration statistics to further evaluate the small-scale isotropy in rotating Rayleigh-Bénard convection.

The acceleration fluctuations are reported elsewhere [39]. Here, we focus on the values of the constant $a_{0}$. Generally, the $a_{0}$ values are a function of $\operatorname{Re}_{\lambda}$. We cover different $\operatorname{Re}_{\lambda}$, which are achieved by changing the rotation rate rather than a variation of $\mathrm{Ra}$ or Pr. Table I shows the relation between $\operatorname{Re}_{\lambda}$ and $\mathrm{Ro}$ at the three measurement locations for all eight experiments. The estimated values of $\operatorname{Re}_{\lambda}$ for the measurements at $z=0.8 \mathrm{H}$ and $z=0.975 H$ for Ro $\geq 2.5$ have higher uncertainty due to the subtraction of mean values related with the presence of the LSC, as discussed in Sec. III B 1. The values of $a_{0}$ for different $\operatorname{Re}_{\lambda}$ are plotted in Fig 7. Filled symbols are data of the current study and open symbols represent the data from previous studies [55-58]. The black line is a fitted curve to the data in literature, taken from [56].

TABLE I. $\operatorname{Re}_{\lambda}$ as a function of Ro at the three measurement locations for all eight experiments.

\begin{tabular}{llllllllll}
\hline \hline & \multicolumn{1}{c}{ Ro } \\
Position & 0.1 & 0.2 & 0.5 & 1 & 2.5 & 3 & 5 & $\infty$ \\
\hline Center & 8 & 10 & 15 & 21 & 38 & 39 & 34 & 39 \\
$z=0.8 H$ & 10 & 13 & 17 & 21 & $30^{\mathrm{a}}$ & $30^{\mathrm{a}}$ & $27^{\mathrm{a}}$ & $28^{\mathrm{a}}$ \\
$z=0.975 H$ & 11 & 14 & 18 & 21 & $25^{\mathrm{a}}$ & $24^{\mathrm{a}}$ & $24^{\mathrm{a}}$ & $27^{\mathrm{a}}$ \\
\hline \hline
\end{tabular}

${ }^{a}$ higher uncertainty due to the presence of the LSC.

The calculated $a_{0}$ at the center shows no appreciable dependence on direction, see the blue squares and red circles in Fig. 7. For Ro $=0.1$ the horizontal component is smaller, however, the difference is within the experimental uncertainty $(\sim 15-25 \%)$. For $z=0.8 H$, a difference between horizontal and vertical directions is noticeable, see the cyan triangles and green six-point stars in Fig. 7, which is an indication that the small scales are becoming anisotropic. The $a_{0}$ values are still in the range of the predicted values for HIT. For $z=0.975 H$, the values of $a_{0}$ for horizontal and vertical directions are significantly different for Ro $\lesssim 2.5$, see yellow pluses and dark red diamonds in Fig. 7. Furthermore, $a_{0}$ for the horizontal component is much larger than what is expected for HIT, while for the vertical direction it is smaller than expected. For $R_{0} \geq 2.5$, the values of $a_{0}$ for horizontal and vertical directions collapse on top of each other and they are close to the predicted value for HIT. However, the uncertainty in the estimation of $\operatorname{Re}_{\lambda}$ is high and should be kept in mind.

Fig. 8 shows $a_{0}$ as a function of Ro. The symbols and colors are the same as in Fig. 7. The values of horizontal and vertical $a_{0}$ decrease with decreasing Ro for the measurements at the cell center for Ro $<2.5$ (red circles and blue squares in Fig.8). At $z=0.8 H$, the horizontalcomponent of $a_{0}$ (cyan triangle) does not experience significant changes for Ro $>1$, whereas it slightly decreases for $\mathrm{Ro}_{0}<1$. However, the vertical-component of $a_{0}$ at $z=0.8 H$ decreases with decreasing Ro. The horizontalcomponent of $a_{0}$ measured at $z=0.975 \mathrm{H}$ increases with decreasing Ro due to the formation of vortical plumes near the plate which add a swirling motion in the horizontal plane. However, the vertical component decreases with decreasing Ro. The effects of rotation on the $a_{0}$ values is similar to that of the acceleration fluctuations. For a detailed discussion on the effects of the rotation on the acceleration fluctuations we refer to Ref. [39].

It can be concluded that the large-scale anisotropy is washed away in the dissipative range at the center of the cell, while at $z=0.975 H$ the small scales remain anisotropic with regard to small-scale velocity fluctuations and acceleration variance for $\mathrm{Ro}<2.5$. At $z=0.8 H$, the small-scale flow seems not far from isotropy.

\section{CONCLUSION}

In summary, we have studied the large-scale and smallscale anisotropy in rotating Rayleigh-Bénard convection $(\mathrm{RBC})$ at three different heights from Lagrangian and Eulerian points of view by laboratory experiments and direct numerical simulation (DNS). We have shown that rotation enhances the large-scale anisotropy of velocity fluctuations at the cell center and close to the top plate $(z=0.975 H)$, while at $z=0.8 H$, this large-scale anisotropy is reduced. The nature of anisotropy at the center and and near the top plate $(z=0.975 H)$ are opposite in the sense that at the center the vertical large-scale motions are stronger while at $z=0.975 H$ the horizontal large-scale motions are stronger. Moreover, we have shown based on the inertial range of the second-order structure function that large-sale anisotropy increases at the cell center. However, this tool cannot help us further for the study of large-scale anisotropy at $z=0.8 H$ and $z=0.975 H$.

Furthermore, we have looked into the effects of largescale anisotropy, induced by rotation, on the small scales. In order to evaluate the small-scale isotropy, we have used two approaches. First, the second-order Eulerian structure function (SF) has been analyzed. More specifically, we have examined the experimental dissipation rate 


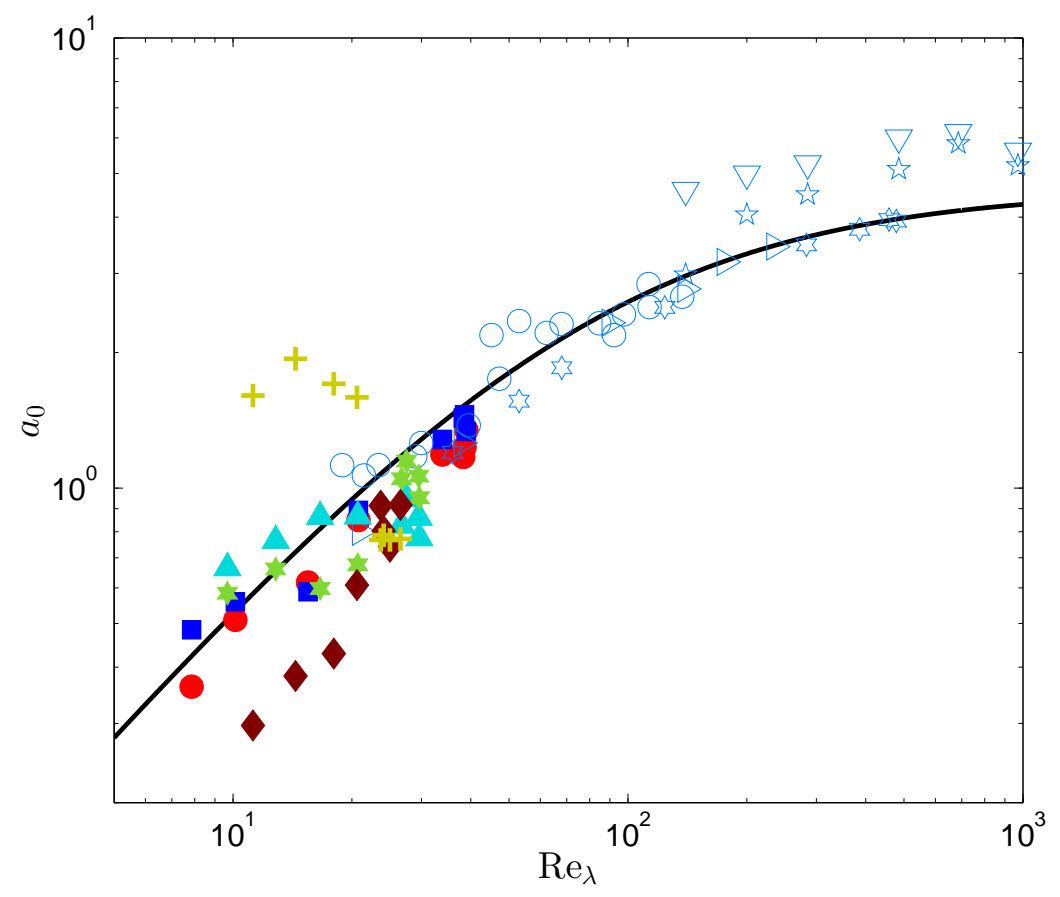

FIG. 7. The Heisenberg-Yaglom constant $a_{0}$ versus $\mathrm{Re}_{\lambda}$. Filled symbols are from the current experiments. Red circles and blue squares are horizontal and vertical component at the center, respectively. Cyan triangles and green six-point stars are horizontal and vertical components at $z=0.8 H$, respectively. Yellow pluses and dark red diamonds are the horizontal and vertical components at $z=0.975 H$, respectively. The estimated error for our data is approximately $15-25 \%$. Open symbols from previous studies; downward-pointing triangles and five-point stars are transverse and axial components, respectively, for experiments on turbulence generated between coaxial counter-rotating disks from [55]; circles for experiments on non-rotating Rayleigh-Bénard convection at the cell center from [56], six-point stars isotropic turbulence DNS data from [57]; and rightpointing triangles isotropic turbulence DNS data from [58]. The black line (taken from [56], $\left.a_{0}=4.6 /\left(1+77.5 / \operatorname{Re}_{\lambda}\right)\right)$ is a fit to all experimental (only axial component from [55]) and numerical data.

based on equations valid for homogeneous and isotropic turbulence (HIT) in the dissipative range of the Eulerian SF. At the center of the cell, the experimental dissipation rates for different rotation rates (with assumption of HIT) agree very well with the dissipation rate calculated using DNS. At $z=0.8 H$, the experimental dissipationrate values follow the trend of the DNS dissipation rate, while the experimental values are generally smaller than the DNS values. At $z=0.975 H$, the experimental values show different trends compared to the DNS data. As for the second approach, we have analyzed the HeisenbergYaglom Lagrangian acceleration constant for different rotation rates. At the cell center, the data shows no dependency on the direction of the acceleration at the cell center. Furthermore, the values of $a_{0}$ for different rotation rates show good agreement with the predicted values for HIT for this range of $\operatorname{Re}_{\lambda}$ at the cell center. At $z=0.8 H$, a difference between horizontal and vertical acceleration constant is noticeable which might be an indication that the small scales are becoming anisotropic. However, the difference is still within the experimental uncertainty. At $z=0.975 H$, the values of $a_{0}$ computed from horizontal and vertical acceleration components are different and they do not match with the predicted values for HIT. Thus, based on both approaches we can conclude that the small scales remain isotropic at the cell center. At $z=0.8 H$, the small-scale flow seems not far from isotropy. However, small anisotropic behaviour is observed. At $z=0.975 H$, small scales are significantly anisotropic.

It can be concluded that the large-scale anisotropy induced by background rotation has hardly any effects on the small scales of the flow in the central region of rotating RBC, while the small-scale velocity and acceleration fluctuations remain anisotropic near the plates(here measured at $z=0.975 H)$.

We hope that this study contributes to the extension of existing theories and arguments for HIT towards realistic flows in which anisotropy is omnipresent. 


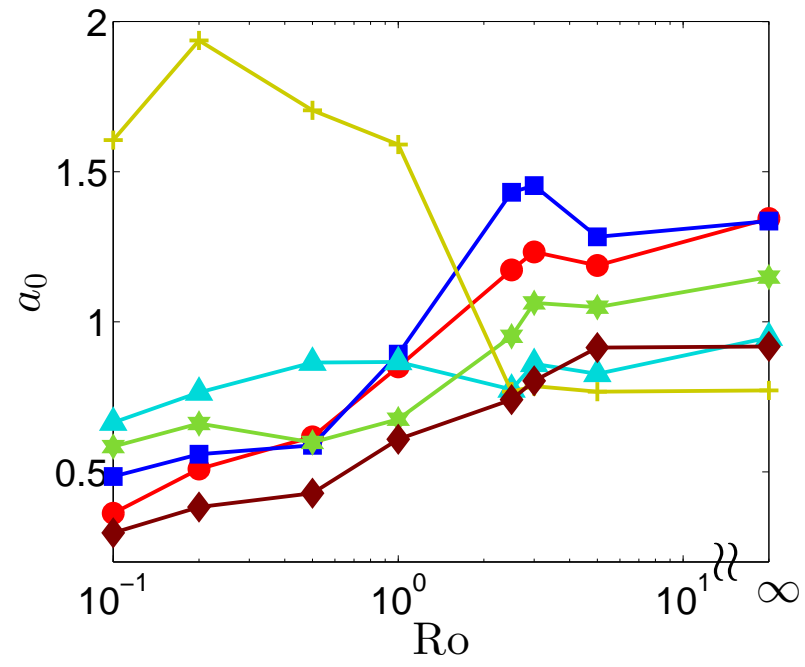

FIG. 8. The Heisenberg-Yaglom constant $a_{0}$ from the experiments versus Ro. Red circles and blue squares represent $a_{0}$ calculated from the horizontal and vertical components a the center, respectively. Cyan triangles and green stars: $a_{0}$ from horizontal and vertical components at $z=0.8 H$, respectively. Yellow pluses and dark red diamonds: $a_{0}$ from horizontal and vertical components at $z=0.975 H$, respectively.

\section{ACKNOWLEDGMENTS}

This work is supported by Stichting voor Fundamenteel Onderzoek der Materie (FOM), Netherlands. The authors gratefully acknowledge the support of the Nederlandse Organisatie voor Wetenschappelijk Onderzoek (NWO) for the use of supercomputer facilities (Cartesius) under Grants No. SH-202 and SH-334.
[1] N. T. Ouellette, H. Xu, M. Bourgoin, and E. Bodenschatz, "Small-scale anisotropy in Lagrangian turbulence," New J. Phys. 8, 102 (2006).

[2] X. Shen and Z. Warhaft, "The anisotropy of the small scale structure in high Reynolds number $\left(R_{\lambda} \sim 1000\right)$ turbulent shear flow," Phys. Fluids 12, 2976-2989 (2000).

[3] Z. Warhaft and X. Shen, "On the higher order mixed structure functions in laboratory shear flow," Phys.Fluids 14, 2432-2438 (2002).

[4] L. Biferale and M. Vergassola, "Isotropy vs anisotropy in small-scale turbulence," Phys. Fluids 13, 2139-2141 (2001).

[5] D. Tordella and M. Iovieno, "Small-scale anisotropy in turbulent shearless mixing," Phys. Rev. Lett. 107, 194501 (2011).

[6] J. Marshall and F. Schott, "Open-ocean convection : Observation, theory, and models." Rev. Geophys. 37, 1-64 (1999).

[7] J. Gascard, A. J. Watson, M. J. Messias, K. A. Olsson, T. Johannessen, and K. Simonsen, "Long-lived vortices as a mode of deep ventilation in the Greenland Sea," Nature 416, 525-527 (2002).

[8] P. Wadhams, J. Holfort, E. Hansen, and J. P. Wilkinson, "A deep convective chimney in the winter greenland sea," Geophys. Research Lett. 29, 1434 (2002).

[9] D. L. Hartmann, L. A. Moy, and Q. Fu, "Tropical convection and the energy balance at the top of the atmosphere," J. Climate 14, 4495-4511 (2001).

[10] M. G. Dunn, "Convective heat transfer and aerodynamics in axial flow turbines," J. Turbomach. 123, 637-686 (2001).
[11] J. P. Johnston, "Effects of system rotation on turbulence structure: a review relevant to turbomachinery flows," Int. J. Rot. Mach. 4, 97-112 (1998).

[12] H. T. Rossby, "A study of Bénard convection with and without rotation," J. Fluid Mech. 36, 309-335 (1969).

[13] B. M. Boubnov and G. S. Golitsyn, "Experimental study of convective structures in rotating fluids," J. Fluid Mech. 167, 503-531 (1986).

[14] F. Zhong, R. E. Ecke, and V. Steinberg, "Rotating Rayleigh-Bénard convection: asymmetric modes and vortex states," J. Fluid Mech. 249, 135-159 (1993).

[15] Y. Liu and R. E. Ecke, "Heat transport scaling in turbulent Rayleigh-Bénard convection: effects of rotation and prandtl number," Phys. Rev. Lett. 79, 2257 (1997).

[16] S. Sakai, "The horizontal scale of rotating convection in the geostrophic regime," J. Fluid Mech. 333, 85-95 (1997).

[17] P. Vorobieff and R. E. Ecke, "Turbulent rotating convection: an experimental study," J. Fluid Mech. 458, 191-218 (2002).

[18] R. P. J. Kunnen, H. J. H. Clercx, and B. J. Geurts, "Enhanced vertical inhomogeneity in turbulent rotating convection," Phys. Rev. Lett. 101, 174501 (2008).

[19] J.-Q. Zhong and G. Ahlers, "Heat transport and the large-scale circulation in rotating turbulent RayleighBénard convection," J. Fluid Mech. 665, 300-333 (2010).

[20] J. J. Niemela, S. Babuin, and K. R. Sreenivasan, "Turbulent rotating convection at high Rayleigh and Taylor numbers," J. Fluid Mech. 649, 509-522 (2010).

[21] R. P. J. Kunnen, B. J. Geurts, and H. J. H. Clercx, "Experimental and numerical investigation of turbulent 
convection in a rotating cylinder," J. Fluid Mech. 642, 445-476 (2010).

[22] E. M. King, S. Stellmach, J. Noir, U. Hansen, and J. M. Aurnou, "Boundary layer control of rotating convection systems," Nature 457, 301-304 (2009).

[23] S. Weiss and G. Ahlers, "Heat transport by turbulent rotating Rayleigh-Bénard convection and its dependence on the aspect ratio," J. Fluid Mech. 684, 407-426 (2011).

[24] R. P. J. Kunnen, Y. Corre, and H. J. H. Clercx, "Vortex plume distribution in confined turbulent rotating convection," Europhys. Lett. 104, 54002 (2014).

[25] R. E. Ecke and J. J. Niemela, "Heat transport in the geostrophic regime of rotating Rayleigh-Bénard convection," Phys. Rev. Lett. 113, 114301 (2014).

[26] R. J. A. M. Stevens, H. J. H. Clercx, and D. Lohse, "Optimal Prandtl number for heat transfer in rotating Rayleigh-Bénard convection," New J. Phys. 12, 075005 (2010).

[27] S. Horn and O. Shishkina, "Toroidal and poloidal energy in rotating Rayleigh-Bénard convection," J. Fluid Mech. 762, 232-255 (2015).

[28] S. Stellmach, M. Lischper, K. Julien, G. Vasil, J. S. Cheng, A. Ribeiro, E. M. King, and J. M. Aurnou, "Approaching the asymptotic regime of rapidly rotating convection: Boundary layers versus interior dynamics," Phys. Rev. Lett. 113, 254501 (2014).

[29] K. Julien, S. Legg, J. McWilliams, and J. Werne, "Rapidly rotating turbulent Rayleigh-Bénard convection," J. Fluid Mech. 322, 243-273 (1996).

[30] R. P. J. Kunnen, H. J. H. Clercx, and B. J. Geurts, "Heat flux intensification by vortical flow localization in rotating convection," Phys. Rev. E 74, 056306 (2006).

[31] S. Schmitz and A. Tilgner, "Heat transport in rotating convection without Ekman layers," Phys. Rev. E 80, 015305 (2009).

[32] K. Julien, A. M. Rubio, I. Grooms, and E. Knobloch, "Statistical and physical balances in low Rossby number Rayleigh-Bénard convection," Geophys. Astrophys. Fluid Dyn. 106, 392-428 (2012).

[33] R. J. A. M. Stevens, H. J. H. Clercx, and D. Lohse, "Heat transport and flow structure in rotating RayleighBénard convection," Eur. J. Mech. B/Fluids 40, 41-49 (2013).

[34] H. G. Maas, A. Gruen, and D. Papantoniou, "Particle tracking velocimetry in three-dimensional flows," Exp. Fluids 15, 133-146 (1993).

[35] N. A. Malik, Th. Dracos, and D. A. Papantoniou, "Particle tracking velocimetry in three-dimensional flows," Exp. Fluids 15, 279-294 (1993).

[36] J. Willneff, A spatio-temporal matching algorithm for $3 D$ particle tracking velocimetry, Ph.D. thesis, Swiss Federal Institute of Technology, Zürich, Switzerland (2003).

[37] J. Willneff, "3D particle tracking velocimetry based on image and object space information," Int. Arch. Photogramm. Rem. Sens. Spatial Inform. Sci. 34, 601-606 (2002).

[38] B. Lüthi, A. Tsinober, and W. Kinzelbach, "Lagrangian measurement of vorticity dynamics in turbulent flow," J. Fluid Mech. 528, 87-118 (2005).

[39] H. Rajaei, P. Joshi, K. M. J. Alards, R. P. J. Kunnen, F. Toschi, and H. J. H. Clercx, "Transitions in turbulent rotating convection: A Lagrangian perspective," Phys. Rev. E 93, 043129 (2016).

[40] R. Verzicco and R. Camussi, "Numerical experiments on strongly turbulent thermal convection in a slender cylindrical cell," J. Fluid Mech. 477, 19-49 (2003).

[41] R. Verzicco and P. Orlandi, "A finite-difference scheme for three-dimensional incompressible flows in cylindrical coordinates," J. Comput. Phys. 414, 402-414 (1996).

[42] G. Ahlers, S. Grossmann, and D. Lohse, "Heat transfer and large scale dynamics in turbulent Rayleigh-Bénard convection," Rev. Mod. Phys. 81, 503 (2009).

[43] R. P. J. Kunnen, H. J. H. Clercx, and B. J. Geurts, "Breakdown of large-scale circulation in turbulent rotating convection," Europhys. Lett. 84, 24001 (2008).

[44] S. Weiss, R. J. A. M. Stevens, Jin-Q Zhong, H. J. H. Clercx, D. Lohse, and G. Ahlers, "Finite-size effects lead to supercritical bifurcations in turbulent rotating Rayleigh-Bénard convection," Phys. Rev. Lett. 105, 224501 (2010).

[45] J. E. Hart, S. Kittelman, and D. R. Ohlsen, "Mean flow precession and temperature probability density functions in turbulent rotating convection," Phys. Fluids 14, 955$962(2002)$.

[46] N. T. Ouellette, H. Xu, and E. Bodenschatz, "Bulk turbulence in dilute polymer solutions," J. Fluid Mech. 629, 375-385 (2009).

[47] R. Ni, S.-D. Huang, and K.-Q. Xia, "Local energy dissipation rate balances local heat flux in the center of turbulent thermal convection," Phys. Rev. Lett. 107, 174503 (2011).

[48] K. R. Sreenivasan, "On the universality of the Kolmogorov constant," Phys. Fluids 7, 2778-2784 (1995).

[49] M. Gibert, H. Xu, and E. Bodenschatz, "Inertial effects on two-particle relative dispersion in turbulent flows," Europhys. Lett. 90, 64005 (2010).

[50] Chao Sun, Quan Zhou, and Ke-Qing Xia, "Cascades of velocity and temperature fluctuations in buoyancy-driven thermal turbulence," Phys. Rev. Lett. 97, 144504 (2006).

[51] A. S. Monin and A. M. Yaglom, Statistical Fluid Mechanics: Mechanics of Turbulence, Vol. 2 (Cambridge, Mass., MIT Press, 1975).

[52] D. Lohse and K.-Q. Xia, "Small-scale properties of turbulent Rayleigh-Bénard convection," Ann. Rev. Fluid Mech. 42, 335-364 (2010).

[53] W. Heisenberg, "Zur statistischen theorie der turbulenz," Zeitschrift für Phys. 124, 628-657 (1948).

[54] A. M. Yaglom, "On the acceleration field in a turbulent flow," C. R. Akad. URSS 67, 795-798 (1949).

[55] A. La Porta, G. A. Voth, A. M. Crawford, J. Alexander, and E. Bodenschatz, "Fluid particle accelerations in fully developed turbulence," Nature 409, 1017-1019 (2001).

[56] R. Ni, S.-D. Huang, and K.-Q. Xia, "Lagrangian acceleration measurements in convective thermal turbulence," J. Fluid Mech. 692, 395-419 (2012).

[57] T. Gotoh and D. Fukayama, "Pressure spectrum in homogeneous turbulence," Phys. Rev. Lett. 86, 3775 (2001).

[58] P. Vedula and P. K. Yeung, "Similarity scaling of acceleration and pressure statistics in numerical simulations of isotropic turbulence," Phys. Fluids 11, 1208-1220 (1999). 